\title{
A Fast and Compact Indexing Technique for Moving Objects
}

\author{
Yonghun Park \\ College of Computing \\ Georgia Institute of Technology \\ Atlanta, GA, USA \\ yhpark1119@gmail.com
}

\author{
Ling Liu \\ College of Computing \\ Georgia Institute of Technology \\ Atlanta, GA, USA \\ ling.liu@cc.gatech.edu
}

\author{
Jaesoo Yoo \\ Dept. Information and \\ Communication Engineering \\ Chungbuk National University, Korea \\ yjs@chungbuk.ac.kr
}

\begin{abstract}
Advances in ubiquitous connectivity and location sensing technology have fuelled a rich collection of location based services (LBSs). Efficient spatial indexing techniques are one of the most effective optimization methods to improve the quality of services. Although a variety of spatial index structures like R-tree family and grid variant index structures have been proposed and deployed in real time location based service provisioning systems, they are known to perform poorly when there is high degree of the skewedness in both density distribution and spatial resolution of mobile objects. First, it is hard to decide the optimal resolution of the grid structure. Second, it is equally hard to build a balanced $R$ Tree like index structure that is effective in handling highly skewed distribution of mobile objects. With these issues in mind, we introduce the concept of spatial order sequences and propose a fast and compact index structure for moving objects by utilizing spatial order sequences through a number of density-conscious optimizations. First, we propose the concept of Ordered-Cell Group $(O C G)$ and design a OCG based grid index structure. Second, we speed up the search efficiency of OCGs by effective compaction of identifiers of OCG cells to maximize the fan-out of index node and decrease the depth of the index. Finally, we develop an efficient query processing algorithm that can effectively utilize OCG cells to speed up the processing of spatial queries. Our experimental results demonstrate the effectiveness of our approach compared to existing index techniques.
\end{abstract}

\section{Introduction}

The ubiquitous connectivity and wide deployment of mobile devices have made location aware computing and location based services (LBSs) a popular information technology in the recent years. LBSs provide mobile users convenient services for retrieval of location-dependent information, such as nearby gas stations, restaurants, transportation, and points of interest, and delivery of content to the mobile users on the road in real time. For example, with LBS, one can find the current location of a moving vehicle, the estimated time to reach a place, or where to meet their friends through location sharing services, such as Foursquare. As the number of mobile devices and the number of location based services grow rapidly, one of the most effective optimization techniques in scaling location based services is to employ efficient spatial indexing structures and algorithms for managing location information of moving objects and querying moving objects in real time.

A spatial index is considered effective if it can handle both retrieval and update of the location information of moving objects in real time within desired response time. However, the index structure that is efficient for querying moving objects may not be effective for frequent location updates and vice versa. Thus, the first requirement in designing an effective spatial index is the capability of handling both frequent location updates and fast processing of location queries in real time. The second requirement in designing an effective spatial index is the capability of providing high performance of LBSs no matter whether the moving objects follow the uniform spatial distribution or skewed spatial distribution. In real world, moving objects move continually and follow skewed spatial distribution. Thus, the result of a query tends to change continously according to the movement of the object such that the query is re-evaluated frequently to keep the accuracy of the result. Furthermore, when the density of mobile objects is changed unpredictably, the index structure should be self-tunable in response to the change of density.

Various spatial index structures have been proposed for managing and querying moving objects. We can categorize them into three classes: the Grid file index structure, the $\mathrm{R}$-tree like indexing structure[1], and the $\mathrm{B}^{+}$-tree variant Index structures based on the Grid file. Unfortunately, most of existing indexing structures and algorithms to date fail to meet both of the above-mentioned requirements.

Concretely, the Gird structure is one of the most popular main memory resident index structures for processing moving object queries and managing moving objects. How- 
ever, Grid index suffers from two limitations. First, it is memory-resident and cannot handle very large datasets and thus it fails to perform when the size of the moving objects exceeds the memory boundary. Second, the performance of grid index is also affected by the relation between the resolution (cell size) and data distribution. If the resolution is too high, the storage cost and query processing cost become worse because most of cells include very small number of objects, with cell-based disk blocks, the storage utilization becomes low. In addition, when the size of cells is too small, the number of cells relevant to a query will be increased, which increases the number of disk $I / O$. If the grid resolution is too large, then the query processing cost becomes worse. This is because when the size of cell is too large, each cell includes too many objects. When a query is overlapped with a large cell, all objects in the cell will be retrieved and evaluated against the query. Therefore, it is a known challenge for grid structure to decide the optimal resolution.

The second and third categories of index structures are disk-resident. Many R-tree variant index structures have been proposed to date, such as $\mathrm{R}^{*}$-tree[2], TPR-tree[3] and $\mathrm{TPR}^{*}$-tree[4]. They are efficient for real time query processing but suffer from high update cost due to frequent splitting and merging of index nodes in the presence of high rates of location updates, which is common for moving objects. To overcome the update performance problems, several research proposals put forward the efforts of employing Grid file index structure, a memory resident indexing technique or employing Grid file in conjunction with $\mathrm{B}^{+}$tree. Representative examples include $\mathrm{B}^{x}$-tree[5], $\mathrm{B}^{\text {dual }}$ tree[6] and $\mathrm{ST}^{2} \mathrm{~B}$-tree[7]. They first overlay the index space with a grid which divids the index space into the same-sized cells. Then, instead of directly using cell based inverted index structure like the Grid file, it will sort the cells using an ordering method such as Hilbert curve or Z-order curve [9]. The cells are used as data nodes in the index structures and are managed by $\mathrm{B}^{+}$-tree index. Only $\mathrm{ST}^{2} \mathrm{~B}$-tree considers various density distributions, but it still suffers from the limitation of deciding the appropriate resolution of the grid.

In this paper, we propose a fast and compact index structure for moving objects, called Ordered-Cell Group index (OCG-index for short), which is efficient even when the density of moving objects is skewed. The $O C G$-index has a number of unique features. First, we introduce the concept of spatial order sequences and the concept of OrderedCell Group. By utilizing the Ordered-Cell Group (OCG), we make better utilization of the storage space by assigning each $O C G$ to a disk block instead of mapping a cell to a disk block. This guarantees the number of objects in a leaf node and enables us to reduce the depth of the index. It leads to save both the storage cost and query processing cost. Second, we design a $O C G$ based grid index struc- ture by utilizing spatial order sequences through a number of density-conscious optimizations. Third, we speed up the search efficiency of $O C G$ s by effective compaction of identifiers of $O C G$ cells to maximize the fan-out of index node and decrease the depth of the index. We observe that there is an opportunity to compress the identifiers of cells using techniques like prefix-tree[8]. By compression, we can increase the fan-out of a node and reduces both the storage cost and query processing cost. Finally, we develop an efficient query processing algorithm that can effectively utilize $O C G$ cells to speed up the processing of spatial queries. Our experimental results demonstrate the effectiveness of our approach compared to existing index techniques.

The rest of paper is organized as follows. Section 2 describes the related work and defines the problems. Section 3 introduces the proposed index structures and section 4 shows the performance evaluation between the proposed index and other index structures. Finally, section 5 concludes the paper and mentions the future work.

\section{Background and Related Work}

Ordering methods, such as Z-order curve and Hilbert curve, are two of the most popular space filling curves, which frequently used for linearization of multidimensional data. A key property of these ordering functions is that it can map multidimensional data to one dimension while preserving the locality of the data points. These ordering methods were originally introduced in [10]. Once the data is sorted according to these ordering, any one-dimensional data structure can be used for indexing the data, such as binary search trees and B-trees. Hilbert curve is proposed to increase the locality of the sequence in comparison with Z-order.

Grid structure divides the data space into cells of the same size according to the resolution. It works like a hash function, which can quickly find the cell in which a moving object resides. Recently, several research efforts use grid structure and cell ordering sequences to store the data of moving objects inside a $\mathrm{B}^{+}$-tree, which shows to improve the update problem of R-tree variant index structures while maintaining good query processing performance for moving objects [5][6][7]. $\mathrm{B}^{x}$-tree[5] is the first index structure using grid structure managed by $\mathrm{B}^{+}$-tree with ordering sequences such as Z-order curve and Hilbert curve. Each cell has identifier on the ordering sequences and is put into $\mathrm{B}^{+}$-tree. $\mathrm{B}^{x}$-tree manage at least two of the $\mathrm{B}^{+}$-tree according to the time sequence to process the future trajectory queries efficiently. It helps to reduce the search boundary of queries. And also, the link of the leaf node to next node in $\mathrm{B}^{+}$-tree is to save the cost traveling index when processing queries. $\mathrm{B}^{\text {dual }}$-tree[6] also has similar approach to $\mathrm{B}^{x}$-tree. However, $\mathrm{B}^{d u a l}$-tree improves the query processing time by 
reducing the search boundary, compared to $\mathrm{B}^{x}$-tree.

Both $\mathrm{B}^{x}$-tree and $\mathrm{B}^{d u a l}$-tree are based on grid structures. However, they do not consider the various distributions of objects. To solve the problem, $\mathrm{ST}^{2} \mathrm{~B}$-tree[7] is proposed. $\mathrm{ST}^{2} \mathrm{~B}$-tree divides the index space according to the object distributions. They manage a global grid structure and the number of objects contained in each cell, and distinguish the regions that have similar density by image processing algorithm. Then, they assign $\mathrm{B}^{x}$-tree for each region with appropriate resolution for its density. However, it cannot handle the environment with highly diverse density distributions. Also $\mathrm{ST}^{2} \mathrm{~B}$-tree suffers from the same limitation of resolution as the Grid file index approach because of the cost of managing and adapting the global grid structure to the density of moving objects.

We define the problems of previous work. The first problem is on deciding an appropriate resolution for a grid structure. $\mathrm{ST}^{2} \mathrm{~B}$-tree tried to solve the problem by clustering the areas having similar density but it has the limitation in the environment with highly skewed objects as we mentioned above. The second problem is on the size for the cell identifiers in a grid structure. By using the identifiers of fixed size, the size of the identifiers depends on the resolution of grid structure regardless of how many empty cells are in the grid. Thus with a grid with high resolution (smaller cell size), the cell identifiers can be quite large and nonnegligible in terms of storage and query processing cost. This observation motivates us to group the cells according to the number of objects contained in the cells, such that we map order cell groups to data nodes of the index instead of cells. By reducing the number of nodes in the index structure, it leads to significant saving in both the storage cost and query processing cost.

\section{The $O C G$-Index Structure and Algorithms}

In this section we first introduce the basic concepts involved in defining our $O C G$-index structure and then describe the query processing algorithm for the $O C G$-index.

\subsection{Ordered Cell Group $(O C G)$}

Given a road network and its grid overlay, we first utilize an ordering method, either Z-order curve or Hilbert curve, to group cells into groups such that each group contains equal or similar number of objects. We call such groups the Ordered Cell Groups (OCGs). We assign each $O C G$ a unique identifier using the list of cell identifiers. The equation 1 computes the set of cells that form an ordered cell group $g$ and the identification of $g$, where $G$ is a set of all $O C G \mathrm{~s}, g . i d$ is the identifier of an instance $g$ of $G, C$ is a set of all cells in grid, c.id is the identifier of a cell $c$, and
$N_{\text {fanout }}$ is the number of fan-out of a data node.

$$
\begin{array}{r}
g_{i} . i d=\left\{c_{n} . i d\left|N_{\text {fanout }} \geq \sum_{j=n}^{m}\right| c_{j} . O \mid, c_{j} \in C,\right. \\
\left.c_{j} . i d<c_{j+1} . i d, 0 \leq n \leq m<|C|\right\}, g_{i} \in G
\end{array}
$$

Figure 1 provides an illustrative example to show how to decide $O C G$ s using Z-order for a grid with the resolution of 4 by 4 . The number of cells is 16 , i.e., $|C|=16$. Figure 1(a) presents the naive approach to indexing moving objects using a grid structure. Figure 1b, Figure 1c, and Figure 1d present a scenario where moving objects are changing their locations at the time $t_{0}, t_{1}$ and $t_{2}$ and how to decide $O C G$ s in each of these three time instances: $t_{0}, t_{1}$ and $t_{2}$. Suppose that the fan-out of data node is 6 . In Figure $1 \mathrm{~b}$, there are 6 objects in whole data space at $t^{0}$. In this cast, there is just one $O C G g_{0}$ in the data structures and the identifier of $g_{0}$ is 0 . At $t^{1}$, an object is added into the cell 2 and $g_{0}$ is split because of overflow. To split a $g_{0}$, we first find the middle object in the ordering sequence and then find the cell overlapped with the middle object. The identifier of the found cell becomes a split key. In this example, the split key is 7 . We create a new $O C G g_{1}$, put all objects lying in the cells whose the identifiers are bigger than 7 into $g_{1}$ and remove these objects from $g_{0}$. Finally, we insert $g_{1}$ into the index. At time $t_{2}$, when a few objects are inserted to $g_{0}$ and overflow occurs at $t_{2}$. Thus, 4 is selected as a split key, and a new $O C G g_{2}$ is created by splitting $g_{0}$ again. The identifiers of $g_{0}, g_{1}, g_{2}$ are 1, 7, 4 respectively. Our $O C G$-index uses $O C G$ s as the index entries, instead of indexing every cell as the entries in the index as done in the naive approach, the number of $O C G \mathrm{~s}$ inserted into the index is much smaller as shown in Figure 1. By utilizing $O C G$ as the unit of indexing, we no longer need to keep every cell identifier in the index, which significantly reduces the size of index and enhances the search efficiency of index.

\subsection{Computing the cell identifier on $O C G$}

In this section we describe how to get an identifier of a grid cell given an Z-order group. In this paper, we use two typical ordering methods, Z-order and Hilbert curve. Hilbert curve follows ' $U$ ' shape while Z-order follows ' $Z$ ' shape. Given that Hilbert curve is proposed to increase the locality of the sequence in comparison with z-order, we first describe how to obtain Z-order curves of a multidimensional space and the properties of $Z$-order curves.

Z-order curve by design splits data space into $2^{n}$ ( $n$ is the binary bits of $\mathrm{Z}$-value) number of equalized and disjoint subspace and the $\mathrm{Z}$-value of a point in multidimensional space is simply calculated by interleaving the binary representations of its coordinate values. The $\mathrm{Z}$-value is used as the name for each subspace. Z-order curve has two important properties: (i) If subspace $A$ encloses subspace $B$, the 


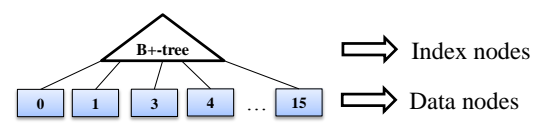

(a) Naive approach

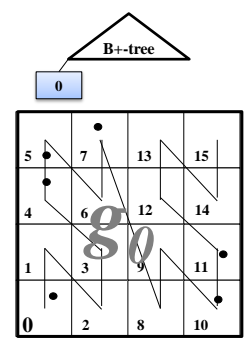

(b) $O C G \mathrm{~s}$ at $t_{0}$

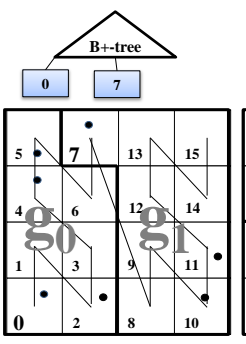

(c) $O C G \mathrm{~s}$ at $t_{1}$

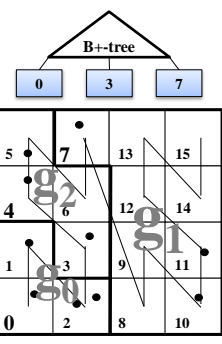

(d) $O C G \mathrm{~s}$ at $t_{2}$
Figure 1: An example of deciding $O C G$ s on Z-order sequence

name of subspace $A$ is a prefix of that of subspace $B$. (ii) If subspace $A$ does not have the same Z-order prefix with subspace $B$, the subspace $A$ does not intersect with subspace $B$.

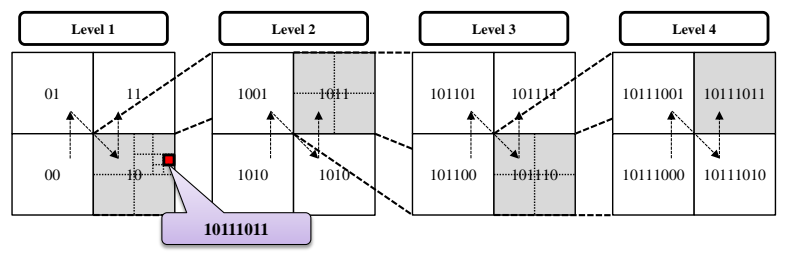

(a) Identifiers of cells on Z-order curve

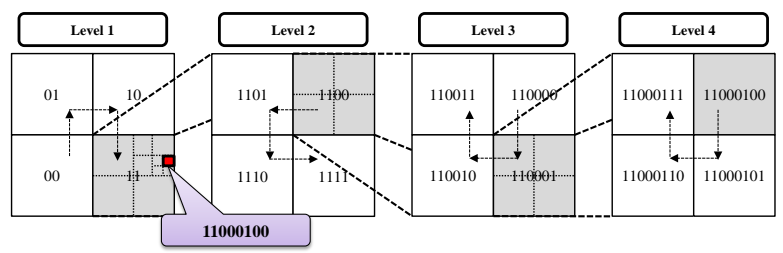

(b) Identifiers of cells on Hilbert-curve

Figure 2: Bit patterns as identifiers of cells on the ordering sequences

We use bit patterns as cell identifiers. An identifier presents the sequence value of a cell on the ordering algorithms. Figure 2 illustrates how the identifier is generated on both Z-order curve and Hilbert curve through the ordering algorithms. It also shows that the same coordinates have different identifier in different algorithms. Both algorithms use 2 additional bits to represent the hierarchical levels of cells in the Grid. In Z-order curve, the front bit and rare bit of the additional bits present $x$ and $y$ coordinates of cells in the data space with $2 \times 2$ resolution respectively, as shown in Figure 2a. While Z-order curve uses always the same shape, in Hilbert curve, the shape is rotated to keep the locality of the sequence according the position of cells as shown in Figure $2 b$. The length of an identifier is determined by the size of the smallest cells in the Grid. We show step by step how a cell identifier is created on Z-order curve and Hilber curve in Figure $2 \mathrm{a}$ and Figure $2 \mathrm{~b}$ respectively. For instance, in Figure 2a we show the cell with 2 bits identifier 10 in level 1 is first split into 4 cells in level 2, each with 4 bits identifier with 10 as Z-order prefix. The cell with identifier 1011 in level 2 continues to be split into 4 cells in level 3, each with 1011 as the Z-order prefix. In level 4, we show that when the cell with identifier 101110 in level 3 is split into 4 cells, their cell identifiers are created by using the same Z-order prefix 101110. Similar prefixing scheme is used for creating cell identifiers on Hilbert-curve.

\subsection{Compression of identifiers}

It is known that one way to decrease the height of the index tree is to increase the fan-out of the index nodes. An advantage of using Z-order prefix on Z-order curve or Hilbert prefix on Hilbert curve to create cell identifiers is the opportunity for compression of identifiers. Given that each $O C G$ is an entry in the $O C G$-index and the identifier of an $O C G$ is composed by the set of cell identifiers of the cells contained in this $O C G$, thus, we can increase the fan-out of the index nodes by compressing the identifiers of the cells.

By looking at the cell identifiers closely, we can observe that a fair number of cells have many zeros in their tails when these cells do not need to be further split while some of their sibling cells continue to be split. Thus, an intuitive approach to compression of cell identifiers is simply to omit the number of 0 on the tail of the identifiers. For example, if an identifier is 011000000 and its size is 64bit (8byte), we present the identifier as 4 bit value 0110 . If we have the value 0110 and the maximum length of the identifier, the decompression is processed by putting the number of 0 on the tail of the values according to the maximum size of identifier. To maximize the fan-out of index nodes, it is important to find a cell that has as many as 0 on the tail of the identifier as a split key when splitting an $O C G$ because of the overflows when more spatial objects enter the spatial region indexed by a given $O C G$. We call the identifier with the maximum number of zeros in its tail the shortest identifier. Formally, we define the shortest identifier as follows.

Definition 1 (Shortest Identifier).

Let $\left[i d_{s}, i d_{e}\right]$ denote the identifier range in a given Grid structure, where $i d_{s}$ and $i d_{e}$ are start and end identifiers of the range respectively, and $L_{\text {sufix }}(i d)$ denote the length of 0 as the suffix of a given identifier $i d$. The shortest identifier, denoted by $I D_{\text {shortest }}$, is an identifier with the maximum length of 0 as its suffix in $\left[i d_{s}, i d_{e}\right]$, formally defined by 
Equation 2:

$$
\begin{aligned}
& I D_{\text {shortest }}\left(i d_{s}, i d_{e}\right)= \\
& \left\{i d_{i} \mid L_{\text {sufix }}\left(i d_{i}\right) \geq L_{\text {sufix }}\left(i d_{j}\right), i d_{s} \leq \exists i d_{i} \leq i d_{e}\right. \\
& \left.\quad i d_{s} \leq \forall i d_{j} \leq i d_{e}\right\}
\end{aligned}
$$

For example, given the identifier range [11010011, 11001000 ], the shortest identifier is 11010000 . Figure 3 shows the algorithm to find the shortest identifier for a given identifier range.

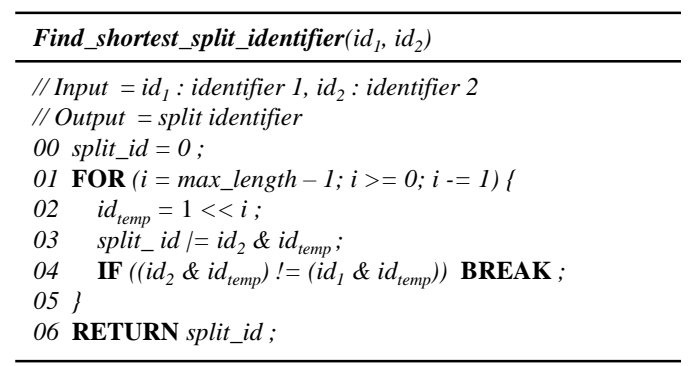

Figure 3: Algorithms to find the shortest split identifier

We use additional information about the length of the identifiers when putting the compressed identifiers into the index nodes. The length information is also used when the identifiers are read from the index nodes. The identifiers are compressed only when they are stored on disk. When processing in memory, we always use the full length of identifiers. To compress identifiers, the length information is predefined as well as the size of the identifiers. Suppose that the size of identifier is 64 bits and the length of an identifier is 4 bits. Each value of 1 in the length information reflects 4bit length of compressed identifier. This means that the identifier is compressed with 4 bits as a unit. A length value 0000 presents that the length of the identifier is 4 and the length value 0001 presents that the length of the identifier is 8 . Both size and length of identifiers are adjustable parameters to be initialized during the system initialization. Given that there is a tradeoff between the length value and the storage cost, it is important to discuss the guidelines for determining the optimal length value of an identifier.

\subsection{Maintaining the index structure}

When an $O C G$ is split due to an overflow over the specified cap of group size in terms of the number of spatial objects, we need to decide a split key in order to split this $O C G$ into two $O C G$ s with smaller spatial regions. To keep the optimal performance of the index structure, we need to consider the compression efficiency and the storage utilization and make a better tradeoff. To achieve better compression efficiency, we want this split key to be as short as possible since it will serve as the prefix. However, to obtain better storage utilization, an $O C G$ should be split into two $O C G \mathrm{~s}$ with equal or similar number of spatial objects. Thus the key is decided by the position of the median object on the ordering sequence. One way to incorporate good compression efficiency into account when choosing the optimal split key is to balance storage utilization with compression efficiency. For example, the split key is decided as short as possible within $1 / 3$ median identifiers of the entries in an $O C G$. If $i d_{s}$ is the identifier of entry at $n / 3$ lowest position on the ordering sequence and $i d_{e}$ is the identifier of entry at $2 n / 3$ lowest position on the ordering sequence, then the split key is decided by $I D_{\text {shortest }}\left(i d_{s}, i d_{e}\right)$ in Equation 2.

In the minimum storage utilization, the first method $(50 \%)$ is better than the second methods (33\%). However, as shown in Section 3.3, the fan-out of index node in the second method is twice more than that in the first method. In the other words, although the storage utilization in the second method is less than in the first method, the number of entries in the second method is in general bigger than the first method. Figure 4 visually shows the difference between the cases. Figure 4a presents the case only considering storage utilization. In the case, according to the increase of objects, the $O C G$ s are split and the split key is decided by the median objects in the ordering sequence. so two groups are made and the difference number of objects between the two groups are equle to or less then 1. Figure $4 \mathrm{~b}$ presents the case considering both storage utilization and compression efficiency.

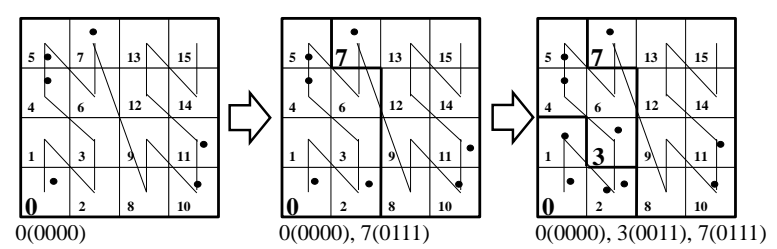

(a) Consider only storage utilization

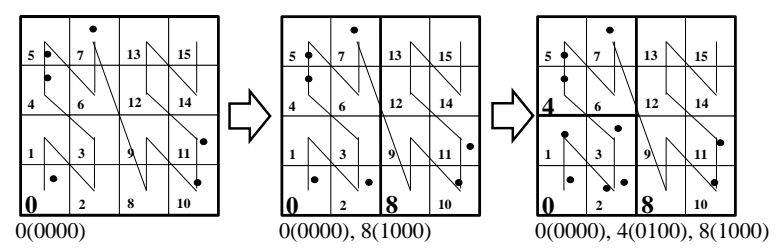

(b) Consider storage utilization and compression efficiency

Figure 4: Index maintenance by tradeoff between storage utilization and compression efficiency

\subsection{Query Processing with $O C G$-index}

In this section we describe the algorithm for processing queries utilizing the proposed index. Due to space constraint, we provide the pseudo code for range query pro- 
cessing algorithm in Figure 5. $k \mathrm{NN}$ queries can be seen as applying a ranking method that sorts the range query results by the ascending order of their distances to the query focal object. In the range query processing algorithm, we first calculate the smallest identifier min_id and the largest identifier $m a x \_i d$ overlapped with the given range in the manner similar to the quad tree search style (line 01 02). The algorithm starts with finding a node including $m i n \_i d$ and retrieving objects that match the query $q$ in the node (line 04 05). Since the index structure is $\mathrm{B}^{+}$-tree, we just use next link of the node to find the next node. If the next node is overlapped with the range given by query $\mathrm{q}$, we continue to search objects for $q$ in the next node. This process continues until the next node is no longer related to $q$ (line 06 09). If the identifier of the next node is larger than max_id, the algorithm terminates. Otherwise, it is continued by finding the next smallest identifier which is an identifier of the cell overlapped with $q$ and the identifier is bigger than the identifier of node searched previously. Equation 3 presents the definition of the next smallest identifier $I D_{\text {next_search }}$, given a query $q$ and the smallest identifier at least id. $c$ denotes a cell in the grid matrix.

$$
\begin{aligned}
& I D_{\text {next }_{\text {search }}}(q, i d)=\left\{c_{i} . i d \mid c_{i} . i d \leq c_{j} . i d,\right. \\
& \left.c_{i} \geq i d, \exists c_{i} \in \text { q.region, } \forall c_{j} \in \text { q.region }\right\}
\end{aligned}
$$

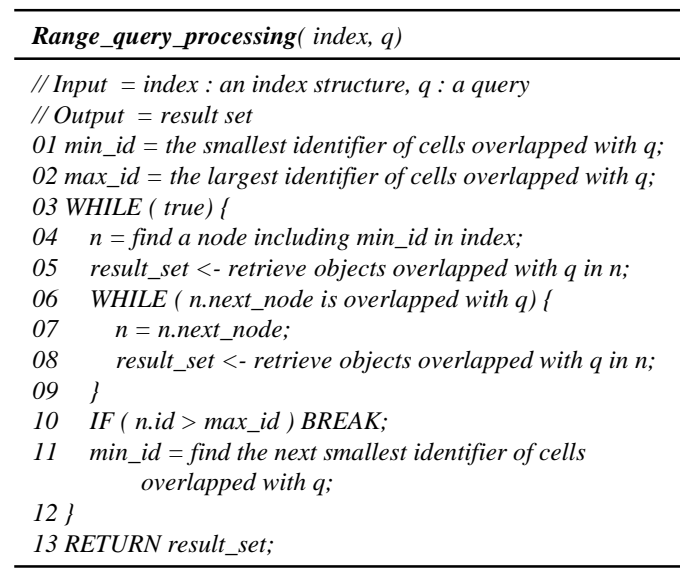

Figure 5: Algorithm for processing range queries

\section{Performance Evaluation}

In this section, we evaluate the performance to show the characteristics of $O C G$-index in various environments. As the ordering method, Z-order and Hilbert curve motheds are used. We also compare the performance according to the compression. The evaluation shows the performance difference in comparison with $\mathrm{R}^{*}$-tree, TPR*-tree and Grid structures. Z_OCG_UNCOM and
$Z_{-} O C G_{-} C O M$ denote the $O C G$-index with Z-order, and $O C G$-index with $\mathrm{Z}$-order with compression, respectively. $H_{-} O C G_{-} U N C O M$ and $H_{-} O C G_{-} C O M$ denote the $O C G$ index with Hilbert curve, and $O C G$-index with Hilbert curve with compression, respectively. For Grid structures, we measured the performance with various resoluation among $512 \times 512(G R I D 512), 256 \times 256(G R I D 256)$, $128 \times 128(G R I D 128), 64 \times 64(G R I D 64)$. We evaluated the storage cost, query processing cost and update cost according to the number of objects, size of queries and velocity of objects. To see the performance according to the density of objects, two different environments are used. Figure 6 shows the environment according to the distribution of objects. Figure 6a and Figure 6b shows random and skewed objects distribution, respectively. As default settings, the size of a disk block assigned to a node is set 2048 bytes and the number of objects inserted into index strucutres is set $1,000,000(1 \mathrm{M})$. The data space is $1000 \times 1000$.

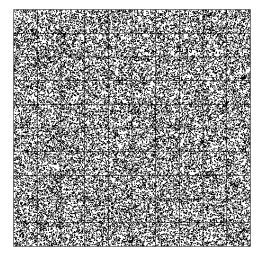

(a) Random distribution

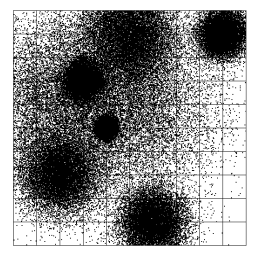

(b) Skewed distribution
Figure 6: Object distribution

We first evaluated the storage cost $O C G$-indexes, $\mathrm{R}^{*}$ tree, TPR*-tree and Grid structures. To measure the storage cost, we count the total number of nodes for each index structures required. Figure 7 presents the comparison. Figure 7a shows the storage cost in random object distribution and Figure $7 \mathrm{~b}$ shows the storage cost in skewed object distribution. It shows that tree-based indexes require simular storage cost while the storage cost of Grid is exponentialy increased according to the increase of resolution. We omitted the storage cost of $Z_{-} O C G_{-} C O M$ and $Z_{-} C O G_{-} U N C O M$ because they have almost the same number of node with regardless of ordering methods.

To show the benefits of $O C G$-indexes, we compared the number of index nodes for each index structures required. Figure 8 shows the total number of index nodes for each index structure requires in random object distribution according to the number of objects. H_OCG_UNCOM reduces about $65 \%$ of index nodes than $\mathrm{R}^{*}$-tree. $H_{-} O C G_{-} C O M$ reduces about $80 \%$ of index nodes than $\mathrm{R}^{*}$-tree and $30 \%$ of index nodes than $H_{-} O C G_{-} U N C O M$. We also measured the average number of entries in an index node to show the storage utilization in terms of index nodes. Figure $8 \mathrm{~b}$ shows the average number of entities. The number of entries depends on the fan-out of node that the index structures allow. 


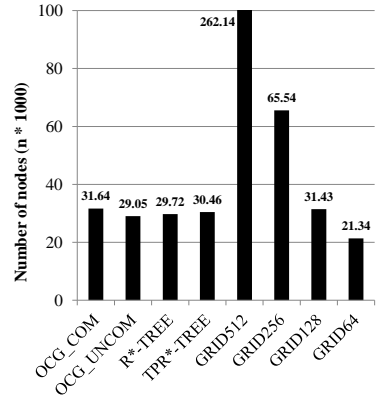

(a) Random distribution

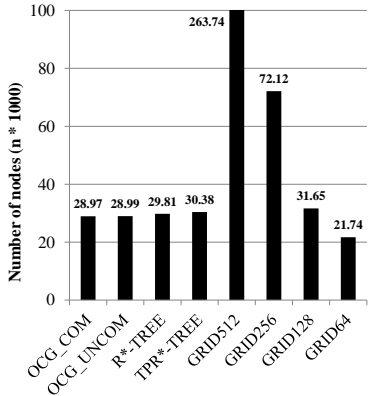

(b) Skewed distribution
Figure 7: Total storage cost

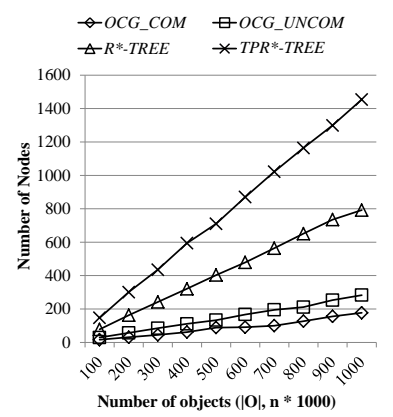

(a) Number of index nodes

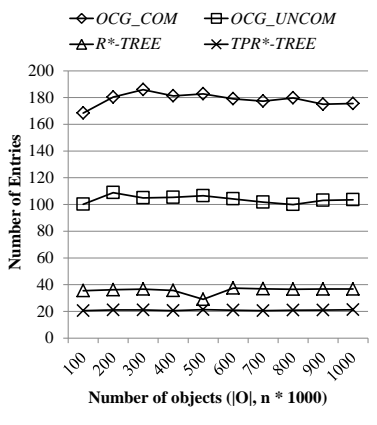

(b) Storage utilization
Figure 8: Storage cost in terms of index nodes

The performance difference is from the size of each entry. Each entry in $O C G$-index includes only the identifier and address of a child node and the length value of the identifier while each entry in $\mathrm{R}^{*}$-tree includes 2 coordinators and the address of chlid node address, and each entry in TPR*-tree additionally includes the two vectors for each dimension. $H_{-} O C G_{-} U N C O M$ and $H_{-} O C G_{-} C O M$ increase the fan-out of index nodes about 4 times and 8 times and than $\mathrm{R}^{*}$ tree, respectively. Since Grid structures does not have index nodes, we did not compare the number of index nodes with Grid structures. The numbers of $Z_{-} O C G_{-} U N C O M$ and $Z_{-} O C G_{-} C O M$ are almost the same as $H_{-} O C G_{-} U N C O M$ and $H_{-} O C G_{-} C O M$. So that we omitted $O C G$-indexes using $\mathrm{Z}$-order in these evaluations.

We compared the range query processing cost according to the size of queries as shown in Figure 9. We performed 100 queries in both random and skewed object environment. GRID256 is chosen to be compared because $256 \times 256$ was the optimal resolution in this evaluation environment, heuristically. As the cost of query processing, we measure the number of disk $I / O$ while processing the queries. In the random distribution environment, $\mathrm{R} *$-tree has the worst performance and GRID256 is the best performance result. all the $O C G$-indexes are in the middle as

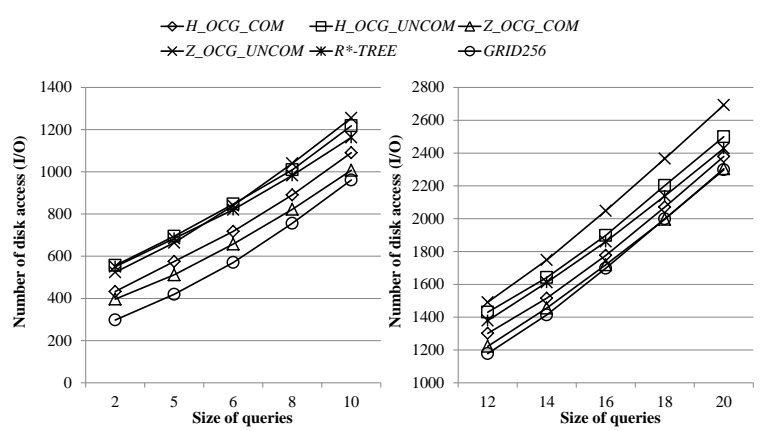

(a) Query processing in random distribution

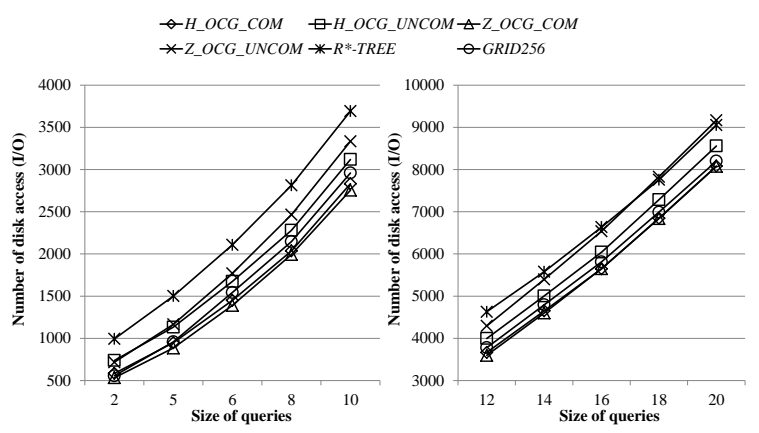

(b) Query processing in skewed distribution

Figure 9: Query processing cost according the size of queries

shown in Figure 9a. However, in the skewed distribution environment, GRID256 becomes worse as shown in Figure 9b. $H_{-} O C G_{-} C O M$ and $Z_{-} O C G_{-} C O M$ become more efficient than $G R I D 256$. In overall, $O C G$-indexes improve the performance of processing queries. $O C G$-indexes without compression save 5 10\% of cost and $O C G$-indexes with compression save $15 \sim 30 \%$ of cost from that of $\mathrm{R}^{*}$ tree. As shown in Figure 9 grid structure is very good in random distribution environment, but it is not efficient in skewed distribution environment.

We compared the query processing cost with TPR*-tree on moving range queries according to the speed of object movements in both random and skewed distribution environments. We performs 100 range queries for $10 \mathrm{sec}$ future from the queries issue and set the size of queris $10 \times 10$. Figure 10 shows the perfomance comparison. In overall, $O C G$-indexes show the better performance than TPR*-tree. $O C G$-indexes without compression save about $5 \sim 10 \%$ of cost and $O C G$-indexes with compression save about $15 \sim 25 \%$ of cost from that of TPR $*$-tree.

For the comparison on update cost, we measured the number disk $I / O$ while update the location of objects according to the speed of object movement in both random and skewed distribution environments. Figure 11 shows the evaluaton of the cost. Since $\mathrm{R}^{*}$-tree is desigined for static 

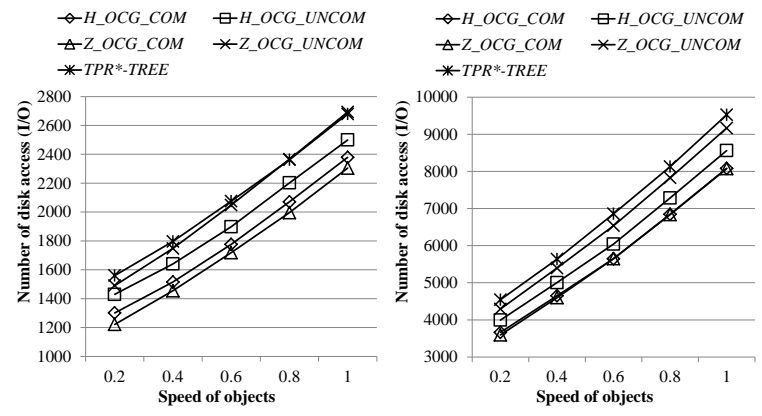

(a) Query processing in ran-(b) Query processing in dom distribution skewed distribution

Figure 10: Moving query processing cost according to the size of queries with TPR*-tree

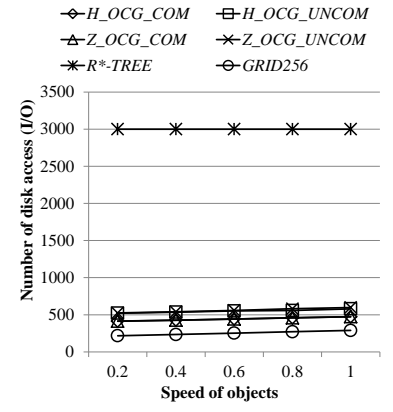

(a) Object update in random(b) Object update in skewed distribution distribution

Figure 11: Update cost according to the speed of objects

objects and sqatial queries, it suffers from the update of objects. Grid structuer shows the best performance on the update of objects' locations. It is because grid structure access the data node directly in hash manner. If the gird structure is optimized the most of update are done with accessing just one data node. The performance of optimized grid can be idle. In overall, $O C G$-indexes are in the middle of $\mathrm{R}^{*}$-tree and grid but they are very close to the idle performance, relatively. In this environment, $O C G$-indexes with compression save about $10 \%$ of update cost than $O C G$-indexes without compression.

\section{Conclusion}

Advances in ubiquitous connectivity and location sensing technology have fuelled a rich collection of location based services (LBSs). Efficient spatial indexing techniques are one of the most effective optimization methods to improve the quality of mobile services. In this paper, we argue that the index structure for LBSs should be efficient for spatial query processing in the presence of frequent location updates. We have introduced the concept of spatial order sequences and the concept of Ordered-Cell Group $(O C G)$ and design a $O C G$ based grid index structure ( $O C G$-index) through a number of density-conscious optimizations. We speed up the search efficiency of $O C G$ s by effective compaction of identifiers of $O C G$ cells to maximize the fanout of index node and decrease the depth of the index. In addition, we develop an efficient query processing algorithm that can effectively utilize $O C G$ cells to speed up the processing of spatial queries. Our experimental results are compared with $\mathrm{R} *$-tree, TPR*-tree, and grid structures. We show that $O C G$-index improves R-tree variant indexes in every aspect we measured and its performance is close to the optimized grid structures.

\section{Acknowledgment}

This work is partially sponsored by grants from NSF CISE NetSE program and SaTC program.

\section{References}

[1] A.Gutmann, "R-trees: A dynamic index structure for spatial searching", In Proc. ACM SIGMOD intl. conf. Management of Data, 1984, pp. 599- 609.

[2] N. Beckmann, H. P. Kriegel, R. Schneider and B. Seeger, ”The $\mathrm{R}^{*}$-tree: an efficient and robust access method for points and rectangles", In Proc. ACM SIGMOD intl. conf. Management of Data, 1990, pp. 322-331.

[3] Saltenis, S., Jensen, C.S., Leutenegger, S.T., Lopez, M.A.: Indexing the positions of continuously moving objects. In: Proceedings of the ACM SIGMOD, pp. 331342 (2000)

[4] Tao, Y., Papadias, D., Sun, J.: The TPR-tree: an optimized spatio-temporal access method for predictive queries. In: Proceedings of the international conference on very large data bases pp. 790801 (2003)

[5] Jensen, C.S., Lin, D., Ooi, B.C.: Query and update efficient $\mathrm{B}^{+}$-tree based indexing of moving objects. VLDB 768779 (2004)

[6] M. L. Yiu, Y. Tao, and N. Mamoulis. The $\mathrm{B}^{\text {dual }}$-tree: Indexing Moving Objects by Space Filling Curves in the Dual Space. VLDB J. 2008.

[7] S. Chen, B. C. Ooi, K. L. Tan and M. A. Nascimento, "The $\mathrm{ST}^{2} \mathrm{~B}$-tree: A Self-Tunable Spatio-Temporal $\mathrm{B}^{+}$-tree Index for Moving Objects", In Proc. ACM SIGMOD intl. conf. Management of Data, 2008, pp. 29-42.

[8] Rudolf Bayer, Karl Unterauer, Prefix B-trees, ACM Transactions on Database Systems (TODS), v.2 n.1, p.11-26, March 1977

[9] Butz, A.R.: Alternative algorithm for Hilberts space-filling curve. IEEE Trans. Comput. C-20(4), 424426 (1971)

[10] G. M. Morton, A computer Oriented Geodetic Data Base; and a New Technique in File Sequencing, Technical Report, Ottawa, Canada: IBM Ltd, 1996. 